\title{
GLOBAL DIMENSION OF TRIANGULAR ORDERS
}

\author{
RICHARD B. TARSY
}

Abstract. The triangular orders of finite global dimension in $n \times n$ matrices over the quotient field of a DVR are found and a bound is given for their dimensions.

1. Introduction. Recently there have appeared examples ([2], [7]) of orders of arbitrarily high finite global dimension in matrix algebras over the quotient field of a DVR, showing that the previously suspected bound of one is exceeded. These examples are of orders of a special type which we call triangular. In this note we find all the triangular orders of finite global dimension in $n \times n$ matrices over the quotient field of a DVR and give a bound for their dimensions. We also observe that the finite finitistic global dimensions of tiled orders in $n \times n$ matrices must be bounded.

2. Definitions, notation, and remarks. We shall be concerned with orders in $n \times n$ matrices, $M_{n}(K)$, over the quotient field, $K$, of a DVR, $R$, with maximal ideal $(t)$. By a tiled order we shall mean an order which can be represented as $\left(\left(t^{a(i, j)}\right)\right)$, i.e. the set of all matrices in $M_{n}(K)$ whose $i, j$ th entry is an element of the ideal $\left(t^{a(i, j)}\right)$ of $R$, $a(i, j)$ an integer. That these are orders implies that $a(i, i)=0$. A triangular order is a tiled order with $a(i, j)=0$ for $i>j$.

The condition that $\left(\left(t^{a(i, j)}\right)\right)$ be a triangular order is simply that $a(i, j)=0$ if $i \geqq j, a(i, j) \geqq a(k, j)$ if $i \geqq k, a(i, j) \geqq a(i, k)$ if $j \geqq k$, and $a(i, k)+a(k, j) \geqq a(i, j)$ if $i \leqq j \leqq k$. It is easily seen then that there are positive integers $m_{1}, \cdots, m_{r}$ with $\sum m_{i}=n$ such that $a(i, j)$ is constant as $i$ runs from $m_{k}$ to $m_{k+1}-1$ and $j$ runs from $m_{p}$ to $m_{p+1}-1$, $k, p=1, \cdots, r$. Thus, by using block notation, we may without loss of generality assume that $a(i, i+1)>0$. We shall suppress further mention of the block convention, assuming all blocks to be $1 \times 1$ for the sake of simplicity. Any changes necessary for the case of larger blocks will be clear.

If $P$ is an order $J(P)$ will denote the Jacobson radical of $P$. If $M$ is a left (right) $P$ module $\operatorname{ld}_{P}(M)\left(\operatorname{rd}_{P}(M)\right)$ will denote the left (right) projective dimension of $M$. GD $(P)$ will denote the global dimension of $P$, the same on left and right since $P$ is left and right Noetherian. Recall also that there is no difference between the projective and weak dimensions of finitely generated $P$ modules.

Received by the editors July $23,1970$.

AMS 1970 subject classifications. Primary 18G20; Secondary 16A62.

Key words and phrases. Global dimension, tiled order, discrete valuation ring. 
We remark in passing that using the known behavior of global dimension with respect to localization and completion all that follows can easily be generalized to the appropriate kinds of tiled orders in central simple algebras over the quotient field of a Dedekind domain.

\section{Main theorem.}

ThEOREM 1. Let $P=\left(\left(t^{a(i, j)}\right)\right)$ be a triangular order in $M_{n}(K)$. GD $(P)$ is finite if and only if $a(i, j) \leqq j-i$ for $i<j$. In this case $\operatorname{GD}(P) \leqq 2$ $+2(n-3)$ if $n \geqq 3$ and $\mathrm{GD}(P) \leqq n-1$ if $n \leqq 3$.

REMARKs. The condition $a(i, j) \leqq j-i$ may be interpreted simply as $a(i, j)-a(i, j-1) \leqq 1$ and $a(i, j)-a(i-1, j) \leqq 1$.

The proof of the theorem depends on several preliminary results which follow.

Lemma 1. Let $S$ be a left Noetherian ring and I a two-sided ideal in $J(S)$. If $M$ is a finitely generated left $S$ module such that $\operatorname{Tor}_{p}^{S}(S / I, M)$ $=0($ all $p>0)$ then $\operatorname{ld}_{S}(M)=\operatorname{ld}_{S / I}(M / I M)$.

Proof. Lemma $1^{\prime}$ in [6].

Lemma 2. If $S$ is a left Noetherian ring and $I$ is a two-sided ideal in $J(S)$ then left $\mathrm{GD}(S) \leqq$ weak $\operatorname{rd}_{S}(S / I)+$ left $\mathrm{GD}(S / I)$.

Proof. Theorem 1 in [6].

Lemma 3. Suppose $S$ is a ring and I is a left projective two-sided ideal with $I^{m}=I^{m+1}$. Then for any $S / I$ module $M \operatorname{ld}_{S I I}(M) \leqq \operatorname{ld}_{S}(M)+2 m-2$. Thus if $1 \mathrm{GD}(S)$ is finite so is $\mathrm{GDD}(S / I)$.

Proof. Theorem 1 in [1].

Lemma 4. Suppose $P$ is an order in $M_{n}(K)$ and $I$ is a two-sided ideal of $P$. There exists an $m$ such that $I^{m}=I^{m+1}$ if and only if $I \nsubseteq J(P)$.

PRoof. If there exists such an $m$ then certainly $I \nsubseteq J(P)$ for $J(P)^{r}$ $C t P$ for a suitable $r$.

On the other hand if $I \nsubseteq J(P)$ then $J(P) \underset{\ddagger}{\subsetneq} I+J(P)$. By Lemma 3.2 of [3] there is an $m$ such that $(I+J(P))^{m}=(I+J(P))^{m+1}$. Multiplying out makes it clear that $I^{m}+J(P)=I^{m+1}+J(P)$ and the conclusion follows from Lemma 2.4 of [3].

Let $P$ be an order in $M_{n}(K)$ and $I$ a left ideal of $P$ that spans $M_{n}(K)$ over $K$. Observe that $\operatorname{Hom}_{p}(I, P)=\left\{x \in M_{n}(K) \mid I x \subset P\right\}$. The dual basis lemma for projective modules then yields 
Lemma 5. $I$ is a left projective $P$ module if and only if 1 $\in \operatorname{Hom}_{P}(I, P) I$.

Proof of Theorem 1. Assume first that $\mathrm{GD}(P)$ is finite. The twosided ideal $I$ which is $P$ with $\left(t^{a(1,1)}\right)=R$ replaced by $\left(t^{a(1,2)}\right)$ is left projective by Lemma 5. Also since $J(P)$ is just $P$ with the diagonal entries replaced by $(t)$ 's, $I \nsubseteq J(P)$. Thus by Lemma $4 I$ fulfills the conditions of Lemma 3 , and so $\operatorname{GD}(P / I)$ is finite. But $P / I$ $\cong R /\left(t^{a(1,2)}\right)$, whence $a(1,2)=1$. Similarly replacing $\left(t^{a(n, n)}\right)$ by $\left(t^{a(n-1, n)}\right)$ yields a projective ideal and so $a(n-1, n)=1$. Proceeding as above, successively replacing $\left(t^{a(i, i)}\right)$ by $\left(t^{a(i, i+1)}\right), i=2, \cdots, n-2$, shows that $a(i, i+1)=1, i=1, \cdots, n-1$. Using induction on $j-i$,

$$
a(i, j) \leqq a(i, i+1)+a(i+1, j) \leqq 1+j-i-1=j-i .
$$

Now suppose $a(i, i+1)=1, i=1, \cdots, n-1$. We proceed by induction assuming $n>3$, since the cases $n \leqq 3$ are covered in Theorems 7 and 12 of [7]. Let $I$ be the two-sided ideal

$$
\left[\begin{array}{cccc}
\left(t^{a(1, n)}\right) & \left(t^{a(1, n)}\right) & \cdots & \left(t^{(1, n)}\right) \\
\left(^{a(2, n)}\right) & \left(t^{a(2, n)}\right) & \cdots & \left(t^{a(2, n)}\right) \\
\vdots & & & \\
(t) & (t) & \cdots & (t) \\
R & R & \cdots & (t)
\end{array}\right]
$$

$I$ is right projective, for the first $n-1$ rows of $I$ are each just the last row of $P$ multiplied by a power of $t$, and the last row of $I$ is the $(n-1)$ th row of $P$. Clearly $I \subset J(P)$. By Lemma $2, \mathrm{GD}(P) \leqq 1$ $+\mathrm{GD}(P / I)$. Now $P / I \cong Q / L \oplus R /(t)$ (ring sum) where $Q$ is a triangular order in $M_{n-1}(K)$ and $L$ is the ideal of $Q$ which is $I$ with the last row and column deleted. We have $\mathrm{GD}(P / I)=\mathrm{GD}(Q / L)$, and by induction $\mathrm{GD}(Q) \leqq 2+2(n-4)$. It remains to compute $\mathrm{GD}(Q / L)$, which may be done by computing $\operatorname{ld}_{Q / L} J(Q / L)$, since $Q / L$ is a finite dimensional algebra over $R /(t) ; \mathrm{GD}(Q / L)=1+\operatorname{ld}_{Q / L}(J(Q / L))$.

Note that $L \subset J(Q)$ so that $J(Q / L)=J(Q) / L$. Also $L$ is right projective and $J(Q)$ is a submodule of the free $Q$ module, $Q$, from which it follows by the connecting homomorphism theorem [5, Chapter 5], that $\operatorname{Tor}_{p}^{Q}(Q / L, J(Q))=0, p>0$. By Lemma $1, \operatorname{ld}_{Q}(J(Q))=$ $\operatorname{ld}_{Q / L}(J(Q) / L J(Q))$. It is known (see, for example [7]) that $\operatorname{ld}_{Q}(J(Q))$ $=\mathrm{GD}(Q)-1$.

We have the exact sequence $0 \rightarrow L / L J(Q) \rightarrow J(Q) / L J(Q) \rightarrow J(Q) / L$ $\rightarrow 0$. By direct computation $L / L J(Q) \oplus \cdots \oplus L / L J(Q)$ (n sum- 
mands) is isomorphic to $L / t L$ and again by computation $t L=L^{2}$. Thus $\operatorname{ld}_{Q / L}(L / L J(Q))=\operatorname{ld}_{Q / L}\left(L / L^{2}\right)$. But $\operatorname{Tor}_{p}^{Q}(Q / L, L)=0, p>0$, so $\operatorname{ld}_{Q / L}(L / L J(Q))=\operatorname{ld}_{Q}(L) \leqq \mathrm{GD}(Q)-1$. Using the well-known relation of the projective dimensions of modules in an exact sequence we conclude that $\operatorname{ld}_{Q / L} J(Q) / L \leqq \mathrm{GD}(Q)$. Hence, $\mathrm{GD}(Q / L) \leqq \mathrm{GD}(Q)+1$ and $\mathrm{GD}(P) \leqq \mathrm{GD}(Q)+2 \leqq 2+2(n-4)+2=2+2(n-3)$.

THEOREM 2. The number of isomorphism classes of $R /(t)$ algebras $P / t P$ arising as $P$ runs over tiled orders in $M_{n}(K)$ is finite.

Proof. Let $P=\left(\left(t^{a(i, j)}\right)\right)$ be a tiled order in $M_{n}(K) . P$ is a free $R$ module with basis $t^{a(i, j)} e_{i, j}$, where $e_{i, j}$ is the matrix with $i, j$ th entry equal to one and zeroes elsewhere. The residue classes of these basis elements of $P$ form a basis of $P / t P$ over $R /(t)$. Multiplying one by another yields either 0 or another basis element of $P / t P$. Thus $P / t P$ has a basis with algebra structure constants consisting only of 0 's and 1 's. Since there are only finitely many possibilities for such an array there are clearly only finitely many isomorphism classes of $P / t P$ 's arising.

Let $S$ be a ring. Denote by $\operatorname{lfGD}(S)$ the supremum of the projective dimensions of finitely generated left $S$ modules of finite projective dimension.

Corollary 1. As $P$ runs over tiled orders in $M_{n}(K)$ the finite IfGD $(P)$ 's are bounded and, hence, so are the finite $\mathrm{GD}(P)$ 's.

Proof. Standard change of rings results (for example Theorems $C$ and $\mathrm{E}$ of Chapter 4 of $[4])$ show that $\operatorname{lfGD}(P)=\operatorname{lfGD}(P / t P)+1$ and the result follows from Theorem 2 .

\section{BIBLIOGRAPHY}

1. K. L. Fields, On the global dimension of residue rings, Pacific J. Math. 32 (1970), 345-349.

2. - Examples of orders over discrete valuation rings, Math. Z. 111 (1969), 126-130. MR $40 \# 182$.

3. M. Harada, Hereditary orders, Trans. Amer. Math. Soc. 107 (1963), 273-290. MR 27 \#1474.

4. I. Kaplansky, Commutative rings, Allyn \& Bacon, Boston, Mass., 1970.

5. S. Mac Lane, Homology, Die Grundlehren der math. Wissenschaften, Band 114, Academic Press, New York; Springer-Verlag, Berlin, 1963. MR 28 \#122.

6. L. W. Small, $A$ change of rings theorem, Proc. Amer. Math. Soc. 19 (1968), 662 666. MR $36 \# 6460$.

7. R. Tarsy, Global dimension of orders, Trans. Amer. Math. Soc. 151 (1970), 540-544.

University of California, Irvine, California 92664 